\title{
A STUDY OF A COMPETITIVE BASS MODEL WHICH TAKES INTO ACCOUNT COMPETITION AMONG FIRMS
}

\author{
Tohru Ueda \\ NTT Communication Switching Laboratories
}

(Received September 28, 1989; Revised March 20, 1990)

\begin{abstract}
Competition in some telecommunication services has emerged in Japan since deregulation of telecommunication markets in 1985. As a model which can be used to analyze this competition, an extension of the Bass model, a competitive Bass model, is proposed. This model treats not only competition between two firms, but also competition among $n$ firms. For the case of two firms, an equation which must be satisfied, concerning the sales of the two firms, is derived. For the case of $n$ firms, a necessity condition which must be satisfied, concerning the sales of $n$ firms, is formulated as an eigenvalue problem. The dynamics of the diffusion process corresponding to the values of parameters are analyzed. The diffusion process of a telecommunication service into which new common carriers have recently entered is also discussed.
\end{abstract}

\section{Introduction}

The Nippon Telegraph and Telephone Corporation monopolistically controlled Japanese telecommunication services up to 1985. Thereafter, competition in some telecommunication services began emerging. That competition has led to an interest at NTT in forecasting future demand under such competitive circumstances.

A model for analyzing competition between two species is well known among biologists. This model is a natural extension of the logistic model. Among models which represent the innovation diffusion process, the Bass model is as well known as logistic models. The Bass model has been discussed and extended by many authors because it is very simple and explains the diffusion process qualitatively. In this paper, an extension of the Bass model which takes competition into account is proposed.

\section{Exising Models and the Bass Model Which Takes Competition into Account}

The logistic model in the narrow sense is a well known model representing growth, the rate of which is given by the linear function,

$$
(d x / d t) / x=a-b x .
$$

In a model treating competition between two species, which is an extension of the logistic model [1], [9], the existence of a second species deters increases in the species under consideration, that is,

$$
\begin{aligned}
& d x / d t=x(a-b x-c y), \\
& d y / d t=y(d-e y-f x) .
\end{aligned}
$$

where $x$ and $y$ refer to two species, and this model involves the Lotka-Volterra model as a special case $\{e=0 ; d, f<0\}$.

Bass [2] proposed a model in which the probability, $P(T)$, that an initial purchase will be made at $T$ given that no purchase has yet been made is a linear function of the number 
of previous buyers, $Y(T)$, that is,

$$
P(T)=a+(b / m) Y(T)
$$

where $a$ and $b / m$ are constants, $a$ reflects the fraction of all adopters who are innovators, and the product $b / m$ times $Y(T)$ reflects the pressures operating on imitators as the number of buyers increases. Using the likelihood, $f(T)$, of purchase at $T$,

$$
\begin{gathered}
P(T)=f(T) /\{1-F(T)\} \\
Y(T)=m \int_{0}^{T} f(t) d t=m F(T)
\end{gathered}
$$

where

$$
F(T)=\int_{0}^{T} f(t) d t
$$

From Eqs. (2.4), (2.5) and (2.6),

$$
d Y(t) / d t=\{m-Y(t)\}\{a+(b / m) Y(t)\} .
$$

Various extensions of this model have been proposed. A parameter $m$, which is the total amount of purchasing during the period considered in the Bass model, is a function of time $t$ in [13] and a function of prices in [8] and [11]. The second factor in Eq. (2.8) is a function of time $t$ and/or quantity relating to information about goods, such as advertising costs in $[4],[10],[11]$ and [15]. Papers [14] and [5] introduce price, $p(t)$, multiplicatively, that is,

$$
d Y(t) / d t=\exp \{-k \cdot p(t)\}\{m-Y(t)\}\{a+(b / m) Y(t)\} .
$$

In these models there is no competition.

In [6] and [7], competition between two firms is considered and the cumulative sales, $X_{i}$, of firm $i$ is given by

$$
\begin{gathered}
d X_{i} / d t=\left(m-X_{1}-X_{2}\right)\left\{\alpha_{i}\left(1-k p_{i}\right)+\gamma\left(p_{j}-p_{i}\right)\right\} \\
i=1,2 ; j \neq i
\end{gathered}
$$

where

$p_{i}=$ price of firm $i$,

$k=$ industry price-sensitivity parameter,

$\alpha_{i}=$ absolute advantage of one product over another,

$\gamma=$ consumers' perceived degree of substitutability of the two goods.

In this paper, we study an extension of the Bass model applied to a competitive environment. Considering that an extension of Eq. (2.1) is given by Eqs. (2.2) and (2.3), a natural extension of Eq. (2.8) to the case of competitive environment is

$$
\begin{aligned}
& d x / d t=(m-x-y)\left(p_{1}+q_{1} x-r_{1} y\right), \\
& d y / d t=(m-x-y)\left(p_{2}+q_{2} y-r_{2} x\right) .
\end{aligned}
$$

We call this model the competitive Bass model and discuss the diffusion process in the following sections. This model can be extended to treat $n$ services.

In the Bass model, cumulative sales are treated and $d Y(t) / d t$ is positive. The number of subscribers, for existing firms, of such telecommunication services as automobile telephones, portable telephones, radio paging services and so on decreases when new competitors appear. 
For these services, $x$ and $y$ are the numbers of subscribers of an existing firm, $A$ and a competitor, B at time $t$ and are not the cumulative numbers of subscribers. $d x / d t$ and $d y / d t$ can be negative. We must note that parameters, $p_{1}$ and $q_{1}$, may be different from ones, $p_{10}$ and $q_{10}$, in the monopolistic age.

\section{A solution of the Competitive Bass Model}

\subsection{Two firms case}

Equations (2.11) and (2.12) have the same orbit as the differential equation system,

$$
\begin{aligned}
& d x / d \tau=p_{1}+q_{1} x-r_{1} y \\
& d y / d \tau=p_{2}+q_{2} y-r_{2} x
\end{aligned}
$$

that is,

$$
d x / d y=\left(p_{1}+q_{1} x-r_{1} y\right) /\left(p_{2}+q_{2} y-r_{2} x\right),
$$

excluding singular points at which the right-hand sides of Eqs. (2.11) and (2.12) are both zero.

If $q_{1} q_{2}-r_{1} r_{2} \neq 0$, the above equations become to the homogeneous system

$$
\begin{aligned}
& d u / d \tau=q_{1} u-r_{1} \nu \\
& d \nu / d \tau=q_{2} \nu-r_{2} u
\end{aligned}
$$

where

$$
\begin{gathered}
x=u+a \text { and } y=\nu+b, \\
a=\left(p_{1} q_{2}+p_{2} r_{1}\right) /\left(r_{1} r_{2}-q_{1} q_{2}\right), \\
b=\left(p_{2} q_{1}+p_{1} r_{2}\right) /\left(r_{1} r_{2}-q_{1} q_{2}\right) .
\end{gathered}
$$

The characteristic equation of the system given by Eqs. (3.4) and (3.5) is

$$
\lambda^{2}-\left(q_{1}+q_{2}\right) \lambda+q_{1} q_{2}-r_{1} r_{2}=0 .
$$

The discriminant of this equation is

$$
\begin{aligned}
D & =\left(q_{1}+q_{2}\right)^{2}-4\left(q_{1} q_{2}-r_{1} r_{2}\right) \\
& =\left(q_{1}-q_{2}\right)^{2}+4 r_{1} r_{2} .
\end{aligned}
$$

The system given by Eqs. (3.4) and (3.5) is classified on the line of [12] into five cases, where

$$
P=q_{1}+q_{2}, Q=q_{1} q_{2}-r_{1} r_{2} \text { and } D=P^{2}-4 Q .
$$

[I] $Q<0(\Rightarrow D>0)$

[II] $Q>0, D>0$

[III] $D<0, P \neq 0(\Rightarrow Q>0)$

[IV] $D=0, P \neq 0(\Rightarrow Q>0)$

[V] $Q>0, P=0(\Rightarrow D<0)$

For case [I] or for $r_{2} \neq 0$ in case [II], the following relation between $x$ and $y$ holds:

$$
\left|x-a-z_{1}(y-b)\right|^{K}=C_{1}\left|x-a-z_{2}(y-b)\right|^{L}
$$

where

$$
K=\left(q_{2} / r_{2}-z_{1}\right) /\left(z_{2}-z_{1}\right), L=\left(q_{2} / r_{2}-z_{2}\right) /\left(z_{2}-z_{1}\right)
$$


and $z_{1}$ and $z_{2}$ are the roots of equation

$$
r_{2} z^{2}-\left(q_{2}-q_{1}\right) z-r_{1}=0
$$

and the general solution is given by

$$
\begin{aligned}
& u=c_{1} z_{1} \exp \left(\lambda_{1} \tau\right)+c_{2} z_{2} \exp \left(\lambda_{2} \tau\right) \\
& \nu=c_{1} \exp \left(\lambda_{1} \tau\right)+c_{2} \exp \left(\lambda_{2} \tau\right)
\end{aligned}
$$

where $\lambda_{1}$ and $\lambda_{2}$ are the roots of Eq. (3.9) and

$$
\lambda_{i}=q_{2}-r_{2} z_{i}
$$

and $C_{i}, c_{1}$ and $c_{2}$ are arbitrary constants. $\tau$ is transformed into $t$ by

$$
d t / d \tau=1 /(m-x-y) .
$$

For $r_{2}=0$ in case [II], the following relation between $x$ and $y$ holds:

$$
\left|x-a+\frac{r_{1}}{q_{2}-q_{1}}(y-b)\right|^{q_{2}}=C_{2}|y-b|^{q_{1}} .
$$

This relation is also obtained by the exchange of $x$ and $y$ with the same procedure used to obtain Eq. (3.12), if $r_{1} \neq 0$ in case [II].

In case [III], the following relation between $x$ and $y$ holds:

$$
\begin{gathered}
\frac{1}{2} \log \left\{\left(z-\frac{q_{2}-q_{1}}{r_{2}}\right)^{2}+\frac{D}{4 r_{2}^{2}}\right\}-\frac{2 q_{1}}{D^{1 / 2}} \tan ^{-1} \frac{2\left(r_{2} z+q_{1}-q_{2}\right)}{-D^{1 / 2}} \\
=-\log |y-b|+C_{3} .
\end{gathered}
$$

where

$$
z=(x-a) /(y-b)
$$

and when $D<0, r_{2} \neq 0$.

For $r_{2} \neq 0$ in case [IV], the following relation between $x$ and $y$ holds:

$$
\begin{aligned}
\log \mid x & -a-\frac{q_{2}-q_{1}}{2 r_{2}}(y-b) \mid+\frac{\left(q_{1}+q_{2}\right)(y-b)}{2 r_{2}(x-a)+\left(q_{1}-q_{2}\right)(y-b)} \\
=C_{4} . &
\end{aligned}
$$

For $r_{2}=0$ in case [IV],

$$
q_{1}(x-a)=-r_{1}(y-b) \log |y-b| .
$$

In case $[\mathrm{V}]$, the following relation between $x$ and $y$ holds:

$$
r_{2}(x-a)^{2}+2 q_{1}(x-a)(y-b)-r_{1}(y-b)^{2}=C_{5}
$$

where $r_{2} \neq 0$ because $Q=-q_{1}^{2}-r_{1} r_{2}>0$.

$$
\begin{aligned}
& \text { If } q_{1} q_{2}-r_{1} r_{2}=0, r_{1} \neq 0, q_{1}+q_{2} \neq 0 \text { and } q_{1}+r_{1} \neq 0 \\
& \qquad\left(q_{1}+q_{2}\right)\left(q_{2} x+r_{1} y\right)=\left(p_{1} q_{2}+p_{2} r_{1}\right) \log \left|r_{1} y-q_{1} x-k_{1}\right|+C_{6}
\end{aligned}
$$

where

$$
k_{1}=\left(p_{1} q_{1}-p_{2} r_{1}\right) /\left(q_{1}+q_{2}\right)
$$


For the case $\left\{q_{1} q_{2}-r_{1} r_{2}=0\right.$ and $r_{1}=0, q_{1}+q_{2}=0$ or $\left.q_{1}+r_{1}=0\right\}$, the relation which $x$ and $y$ must satisfy can also be obtained, but it is difficult to interpret the parameters qualitatively. Since this case requires a large amount of troublesome discussion, it will not be mentioned further.

\section{$3.2 n$ firms case}

When considering competition among $n$ firms, equations corresponding to Eqs. (2.11) and (2.12) become

$$
\begin{gathered}
d x_{i} / d t=\left(m-\sum_{j} x_{j}\right)\left(a_{i}+\sum_{j} b_{i j} x_{j}\right), \\
i=1,2, \cdots, n,
\end{gathered}
$$

where $b_{i j}(i \neq j)$ is usually negative. From this equation,

$$
\begin{gathered}
d t\left(m-\sum_{j} x_{j}\right)=d x_{i} /\left(a_{i}+\sum_{j} b_{i j} x_{j}\right), \\
i=1,2, \cdots, n .
\end{gathered}
$$

In order to make the integration of the right-hand side in Eq. (3.26) easy, coefficients $\nu_{i}$ are introduced so as to satisfy

$$
\begin{gathered}
\sum_{i} \nu_{i} d x_{i} /\left\{\sum_{k} \nu_{k}\left(a_{k}+\sum_{j} b_{k j} x_{j}\right)\right\} \\
=\sum_{i} \nu_{i} d x_{i} /\left(c+\lambda \sum_{j} \nu_{j} x_{j}\right) .
\end{gathered}
$$

This is formulated as an eigenvalue problem

$$
B^{\prime} \nu=\lambda \nu
$$

where

$$
\nu=\left(\nu_{1}, \cdots, \nu_{n}\right)^{\prime}
$$

and the $(i, j)$-th element of $B$ is $b_{i j}$.

Let the eigenvector corresponding to an eigenvalue $\lambda_{i}$ be $\nu_{i}$. When $\lambda_{k} \neq \lambda_{h}$,

$$
\begin{aligned}
& \nu_{k}^{\prime} d \mathbf{x} /\left(\nu_{k}^{\prime} \mathbf{a}+\lambda_{k} \nu_{k}^{\prime} \mathbf{x}\right) \\
& \quad=\nu_{h}^{\prime} d \mathbf{x} /\left(\nu_{h}^{\prime} \mathbf{a}+\lambda_{h} \nu_{h}^{\prime} \mathbf{x}\right)
\end{aligned}
$$

where

$$
\mathbf{a}=\left(a_{1}, a_{2}, \cdots, a_{n}\right)^{\prime}, \mathbf{x}=\left(x_{1}, x_{2}, \cdots, x_{n}\right)^{\prime} .
$$

Integration of Eq. (3.29) gives a simultaneous equation system

$$
\begin{aligned}
& \left(1 / \lambda_{k}\right) \log \left(\nu_{k}^{\prime} \mathbf{a}+\lambda_{k} \nu_{k}^{\prime} \mathbf{x}\right) \\
& \quad=\left(1 / \lambda_{h}\right) \log \left(\nu_{h}^{\prime} \mathbf{a}+\lambda_{h} \nu_{h}^{\prime} \mathbf{x}\right),
\end{aligned}
$$

in particular, if $n=2$ and $\left\{r_{1} r_{2}-q_{1} q_{2} \neq 0\right\}$,

$$
\begin{aligned}
& \lambda_{i}=r_{2} z_{i}+q_{1} ; L=\lambda_{1} /\left\{r_{2}\left(z_{2}-z_{1}\right)\right\} \\
& K=\lambda_{2} /\left\{r_{2}\left(z_{2}-z_{1}\right)\right\} .
\end{aligned}
$$


This gives the same relation as Eq. (3.12).

\section{Diffusion Process of the Competitive Bass Model}

In this section only cases [I] and [II] are discussed and the following is assumed:

$$
q_{1} q_{2}-r_{1} r_{2} \neq 0 ; m, p_{i}, q_{i}, r_{i}>0 ; z_{1}<0, z_{2}>0
$$

and two firms, $A$ and $B$, compete. Firm $A$ is the first entrant and firm $B$ is a follower. The diffusion process is analyzed, considering whether $\left\{q_{1} q_{2}-r_{1} r_{2}\right\}$ is positive or negative.

From Eq. (3.12), if $y \neq b$,

$$
|y-b|=C_{1}\left|z-z_{1}\right|^{-K}\left|z-z_{2}\right|^{L}
$$

and if $y=b$,

$$
|x-a|=C_{1}
$$

where

$$
z=(x-a) /(y-b) .
$$

We can understand the diffusion process of the competitive Bass model by giving various values to $z$ in Eq. (4.1). Let curves satisfying Eq. (4.1) be $U 1$.

Singular points or equilibrium points where the right-hand sides of Eqs. (2.11) and (2.12) are both zero are

$$
\{m-x-y=0\} \text { and }\{x=a, y=b\} .
$$

Point $\{x=a, y=b\}$ is an isolated singular point. If $U 1$ and $\{m-x-y=0\}$ have a crosspoint, $P$, the points on $U 1$ around $P$ go towards $P . P$ is then called a stable equilibrium point.

From Eqs. (2.11) and (2.12),

$$
(d x+d y) f d t=(m-x-y)\left\{\left(q_{1}-r_{2}\right)(x-a)+\left(q_{2}-r_{1}\right)(y-b)\right\} .
$$

Thus, values $x_{M}$ and $y_{M}$, of $x$ and $y$ which give the maximum of $\{x+y\}$, satisfy

$$
\begin{aligned}
& (x-a) /(y-b)=-\left(q_{2}-r_{1}\right) /\left(q_{1}-r_{2}\right) \text { for } y \neq b \\
& x=a \text { for } y=b
\end{aligned}
$$

or

$$
x+y=m \text {. }
$$

If $y_{M} \neq b$, Eqs. (4.1), (4.3), and (4.5) give

$$
\begin{aligned}
z_{M} & =-\left(q_{2}-r_{1}\right) /\left(q_{1}-r_{2}\right), \\
\left|y_{M}-b\right| & =C_{1}\left|z_{M}-z_{1}\right|^{-K}\left|z_{M}-z_{2}\right|^{L},
\end{aligned}
$$

and

$$
x_{M}-a=z_{M}\left(y_{M}-b\right) .
$$

\subsection{The case where $\left\{q_{1} q_{2}-r_{1} r_{2}>0\right\}$}

In this case, the diffusion process of the competitive Bass model with two competing firms has the following characteristics:

(i) $\quad a, b<0 ; L>0, K>1 ; r_{1} / q_{1}<z_{2}<q_{2} / r_{2}$,

(ii) If $x$ and $y$ are positive, the left-hand side of Eq. (3.12) is not zero. 
(iii) The $\{x, y\}$ satisfying Eq. (3.12) draws two curves in areas divided by a straight line

$$
S 1: x-a-z_{2}(y-b)=0 ; \text { i.e. } z=z_{2} .
$$

(iv) The signs, ( + or -$)$, of $d x / d t$ and $d y / d t$ are determined by the area in which point $(x, y)$ falls, where the first quadrant in the $(x, y)$ plane is divided into six areas by three straight lines:

$$
\begin{aligned}
& S 2: m-x-y=0, \\
& S 3: p_{1}+q_{1} x-r_{1} y=0 ; \text { i.e. } z=r_{1} / q_{1}, \\
& S 4: p_{2}+q_{2} y-r_{2} x=0 ; \text { i.e. } z=q_{2} / r_{2} .
\end{aligned}
$$

Our concern is, however, three areas under $S 2$ and where we must notice that the $\mathrm{x}$-coordinate, $\left\{a-b r_{1} / q_{1}\right\}$, of a crosspoint between $S 3$ and the $x$-axis is negative regardless of the sign of $\left\{q_{1} q_{2}-r_{1} r_{2}\right\}$ because $\left\{a-b r_{1} / q_{1}=-p_{1} / q_{1}\right\}$. (see Fig. 1).

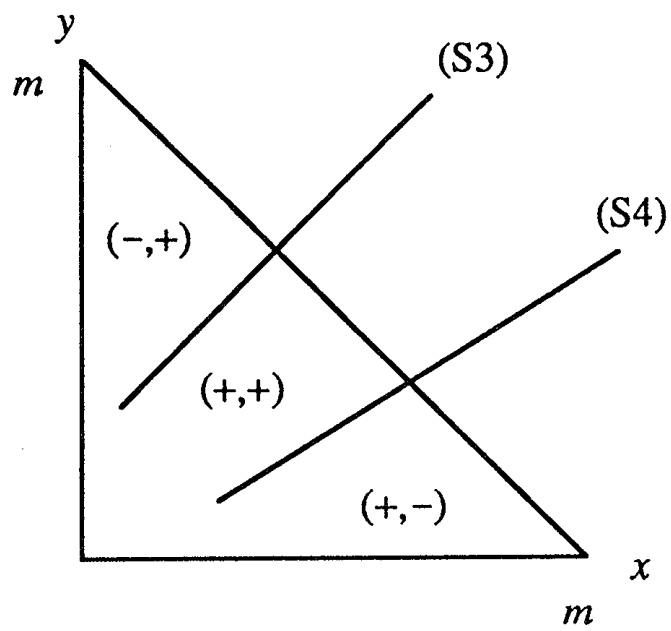

Fig. 1 Symbols of $d x / d t$ and $d y / d t\left(q_{1} q_{2}-r_{1} r_{2}>0\right)$

(v) $\quad S 1$ lies between $S 3$ and $S 4$.

(vi) The diffusion process depends on the value, $x_{0}(>0)$, which firm $A$ takes when firm $B$ is going to enter.

(a) Consider the case $\left\{x_{0}<a-b z_{2}\right\}$. $d x / d t=0$ at $\left(x_{1}, y_{1}\right)$, where

$$
\begin{aligned}
& x_{1}=r_{1}\left(y_{1}-b\right) / q_{1}+a \\
& y_{1}=C_{1}\left(z_{2}-r_{1} / q_{1}\right)^{L} /\left(r_{1} / q_{1}-z_{1}\right)^{K}+b .
\end{aligned}
$$

If $\left\{x_{M}+y_{M} \leq m\right.$ and $\left.y<y_{1}\right\}, x$ and $y$ increase, where $x_{M}$ and $y_{M}$ are given by Eqs. (4.7)-(4.9),

$$
x_{M}+y_{M}=\left\{1-\left(q_{2}-r_{1}\right) /\left(q_{1}-r_{2}\right)\right\}\left(y_{M}-b\right)+a+b
$$

and we must take account of the sign of $\left\{y_{M}-b\right\}$ as seen in Eq. (4.8).

If $\left\{x_{M}+y_{M} \leq m\right.$ and $\left.y>y_{1}\right\}, x$ decreases and $y$ increases.

If $\left\{x_{M}+y_{M}>m\right\}, x$ and $y$ increases until reaching $y=y_{2}$ where $y_{2}$ and $y_{3}\left(y_{2}<y_{3}\right)$ are the $y$-coordinates of crosspoints between $S 2$ and $U 1$. The point 
corresponding to $\left\{y=y_{2}\right\}$ is a stable equilibrium point.

(b) Consider the case $\left\{x_{0}>a-b z_{2}\right\}$. Let the $x$-coordinates of crosspoints between $U 1$ and $S 2$ and between $U 1$ and $S 4$ be $x_{22}$ and $x_{24}$.

Let $x_{24}<x_{22}$. If $x<x_{24}, x$ and $y$ increase, or if $x_{24}<x<x_{22}, x$ increases and $y$ decreases.

Let $x_{22}<x_{24}$, then $x$ and $y$ increase until $x$ reaches $x_{22}$.

The point corresponding to $\left\{x=x_{22}\right\}$ is a stable equilibrium point.

- If $\left\{a-b z_{2}<0\right\}$, the above mentioned (b) is the only possibility considered in the range $\left\{x_{0}>0\right\}$.

[Example 1] In the competitive Bass model

$$
d x / d t=(100-x-y)\{5(x+46.25)-15(y+13.75)\}
$$

and

$$
\begin{gathered}
d y / d t=(100-x-y)\{7(y+13.75)-(x+46.25)\} \\
a=-46.25, b=-13.75, z_{1}=-3, z_{2}=5, K=1.25 \text { and } L=0.25 .
\end{gathered}
$$

When $x_{0}=20$,

$$
C_{1}=275.3, x_{1}=58.3, y_{1}=21.1, x_{M}=50.7, \text { and } y_{M}=34.7 \text {. }
$$

The above mentioned (vi) $-(\mathrm{a})$ is the diffusion process of this model because $\left\{a-b z_{2}=\right.$ $\left.22.5>x_{0}\right\}$. (See Fig. 2)

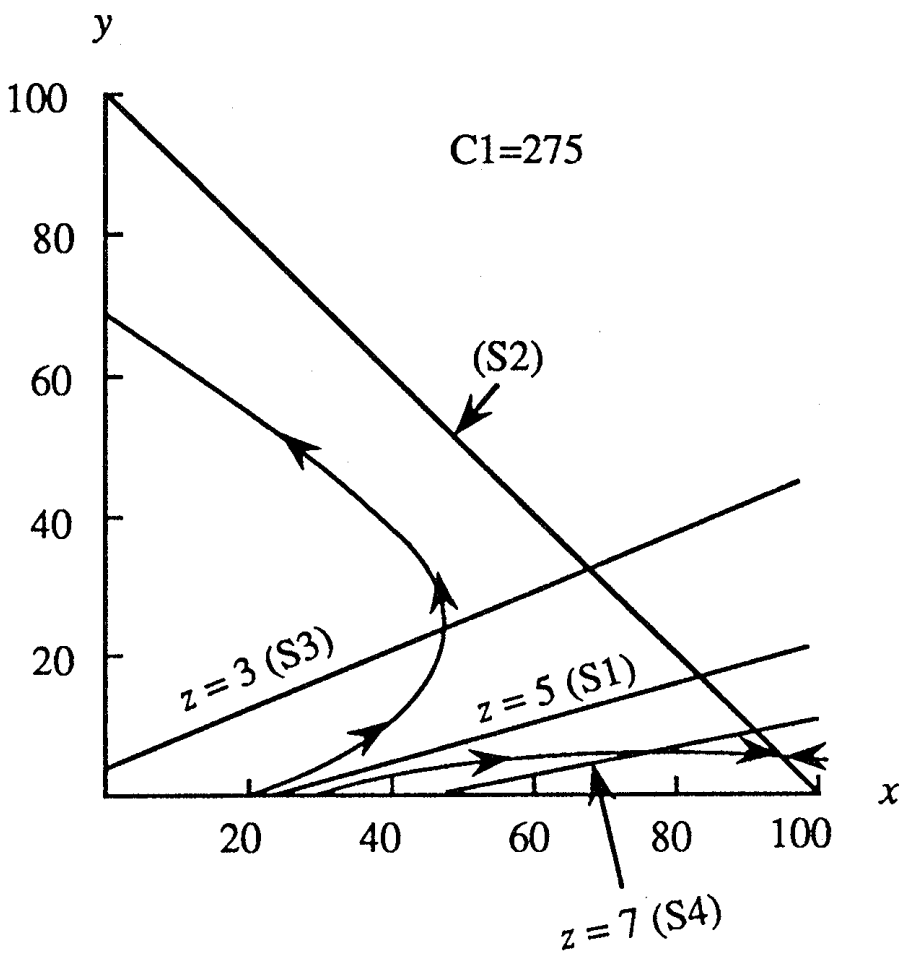

Fig. 2 Diffusion process of Example 1

\subsection{The case where $\left\{q_{1} q_{2}-r_{1} r_{2}<0\right\}$}

In this case, the diffusion process has the following characteristics. 
(i) $\quad a, b>0 ; L<0,0<K<1 ; r_{1} / q_{1}>z_{2}>q_{2} / r_{2}$.

(ii) The range of $\{x, y\}$ satisfying Eq. (3.12) is divided into four areas by straight lines $S 1$ and

$$
T 1: x-a-z_{1}(y-b)=0 ; \text { i.e. } z=z_{1} \text {. }
$$

(iii) (The signs of $d x / d t$ and $d y / d t$ ) are shown in Figs. 3 and 4, where the first quadrant in the $(x, y)$ plane is divided into seven areas by $S 2, S 3$, and $S 4$, but our concern is four areas in Fig. 3 and three areas in Fig. 4 under $S 2$.

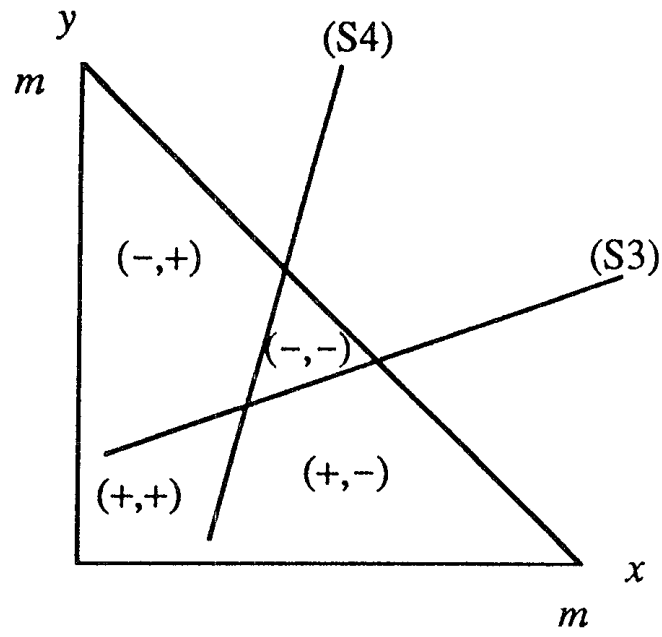

Fig. 3 Symbols of $d x / d t$ and $d y / d t\left(q_{1} q_{2}-r_{1} r_{2}<0, a+b<m\right)$

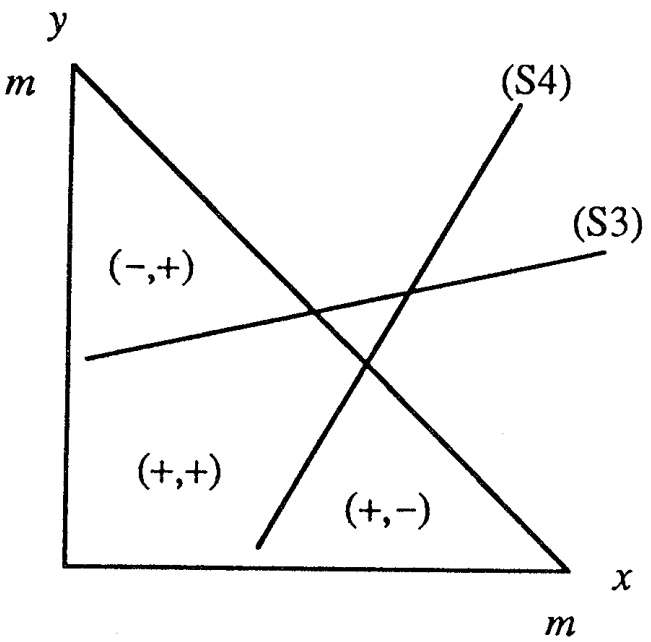

Fig. 4 Symbols of $d x / d t$ and $d y / d t\left(q_{1} q_{2}-r_{1} r_{2}<0, a+b<m\right)$

(iv) $\quad S 1$ lies between $S 3$ and $S 4$.

(v) The diffusion process depends on a value, $x_{0}$ which firm $A$ takes when firm $B$ is going to enter.

(a1) Consider the case $\left\{x_{0}<a-b z_{2}\right.$ and $\left.x_{M}+y_{M} \leq m\right\}$. If $\left\{y<y_{1}\right\}, x$ and $y$. increase, where

$$
\left|y_{1}-b\right|=C_{1}\left(r_{1} / q_{1}-z_{1}\right)^{-K^{K}}\left(r_{1} / q_{1}-z_{2}\right)^{L} .
$$

If $\left\{y>y_{1}\right\}, x$ decreases and $y$ increases.

(a2) If $\left\{x_{0}<a-b z_{2}\right.$ and $\left.x_{M}+y_{M}>m\right\}, x$ and $y$ increase until reaching $y=y_{2}$ where $y_{2}$ and $y_{3}\left(y_{2}<y_{3}\right)$ are the $y$-coordinates of crosspoints between $S 2$ and $U 1$. The point corresponding to $\left\{y=y_{2}\right\}$ is a stable equilibrium point.

(b1) If. $\left\{a-b z_{2}<x_{0}<a-b q_{2} / r_{2}\right.$ and $\left.x<x_{4}\right\}, x$ and $y$ increase, where

$$
\begin{aligned}
& x_{4}=q_{2}\left(y_{4}-b\right) / r_{2}+a ; \\
& \left|y_{4}-b\right|=C_{1}\left(z_{2}-q_{2} / r_{2}\right)^{L} /\left(q_{2} / r_{2}-z_{1}\right)^{K} .
\end{aligned}
$$

If $\left\{a-b z_{2}<x_{0}<a-b q_{2} / r_{2}\right.$ and $\left.x_{4}<x\right\}, x$ increases and $y$ decreases.

(b2) If $\left\{a-b q_{2} / r_{2}<x_{0}\right\}$, the monopoly continues.

When $\left\{a-b z_{2}\right\}$ or $\left\{a-b q_{2} / r_{2}\right\}$ is negative, conditions which involve them are neglected and only the area where $0<x_{0}$ is relevant.

[Example 2] In the competitive Bass model

$$
d x / d t=(100-x-y)\{3(x-85)-15(y-25)\}
$$


and

$$
d y / d t=(100-x-y)\{5(y-25)-2(x-85)\}
$$

$a=85, b=25, z_{1}=-2.284, z_{2}=3.284, K=0.859$, and $L=-0.141$. When

$$
x_{0}=70, C_{1}=71.37, \text { and } a-b q_{2} / r_{2}=22.5<x_{0},
$$

then, the diffusion process is $(\mathrm{v})-(\mathrm{b} 2)$. The $x$-coordinates of the crosspoints between $U 1$ and the $x$-axis are $x_{02}=1.7$ and $x_{03}=4.2$, in addition to $x_{01}=70$. As seen in Fig. 5, when firm $Y$ is going to enter the market,

(i) if the value of $x_{0}$ is $x_{01}$, firm $B$ cannot enter,

(ii) if the value of $x_{0}$ is $x_{02}$, firm $B$ survives in the end,

(iii) if the value of $x_{0}$ is $x_{03}$, firm $A$ survives in the end.

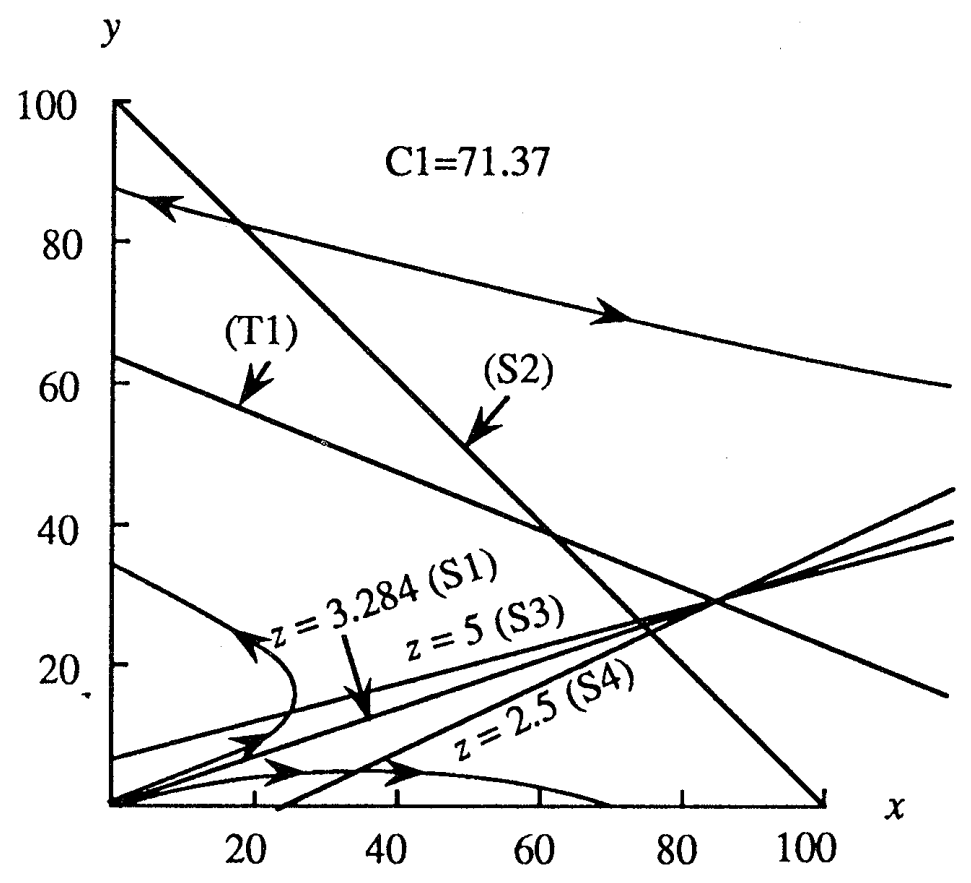

Fig. 5 Diffusion process of Example 2

\section{Application Example}

The diffusion process of a telecommunication service, into which new common carriers (NCC) have recently entered, is discussed. Let the number of subscribers at the end of the fiscal year in which NTT began to offer its service be the unit of measurement. Until the NCCs entered, the growth rate of subscribers was nearly constant for about ten years, excluding the first several years, and we estimated the total demand to be 850 , using another method. These facts gave the following model:

$$
d x_{t} / d t=2 \times 10^{-4} x_{t}\left(850-x_{t}\right)
$$

where $x_{t}$ and $y_{t}$ are the number of subscribers for NTT and the NCCs in year $t$. This model is both a logistic model and a Bass model which does not have any innovation effects.

We can use only 12 months' worth of data, since the NCCs entered the market, i.e., $X_{T}$ and $Y_{T}$ (month $T=1,2, \cdots, 12$ ), for NTT and the NCCs, respectively. This gives the approximation,

$$
x_{t+1}-x_{t}=12\left(X_{T+1}-X_{T}\right),
$$


Set

$$
y_{t+1}-y_{t}=12\left(Y_{T+1}-Y_{T}\right)
$$

$$
S_{T}=X_{T}+Y_{T}
$$

If the same growth continued before and after the entrance of the NCCs, from Eqs. (5.1)-(5.3), the relation

$$
\left(S_{T+1}-S_{T}\right) / S_{T}=2 \times 10^{-4}\left(850-S_{T}\right) / 12
$$

would hold approximately. As seen in Fig. 6, the value of the left side of Eq. (5.4) tends to be larger than that of the right side. The entrance of the NCCs may create new demand. Taking into account all these facts, the relation

$$
D_{T} \equiv\left(S_{T+1}-S_{T}\right) / S_{T}=2.6 \times 10^{-4}\left(1000-S_{T}\right) / 12
$$

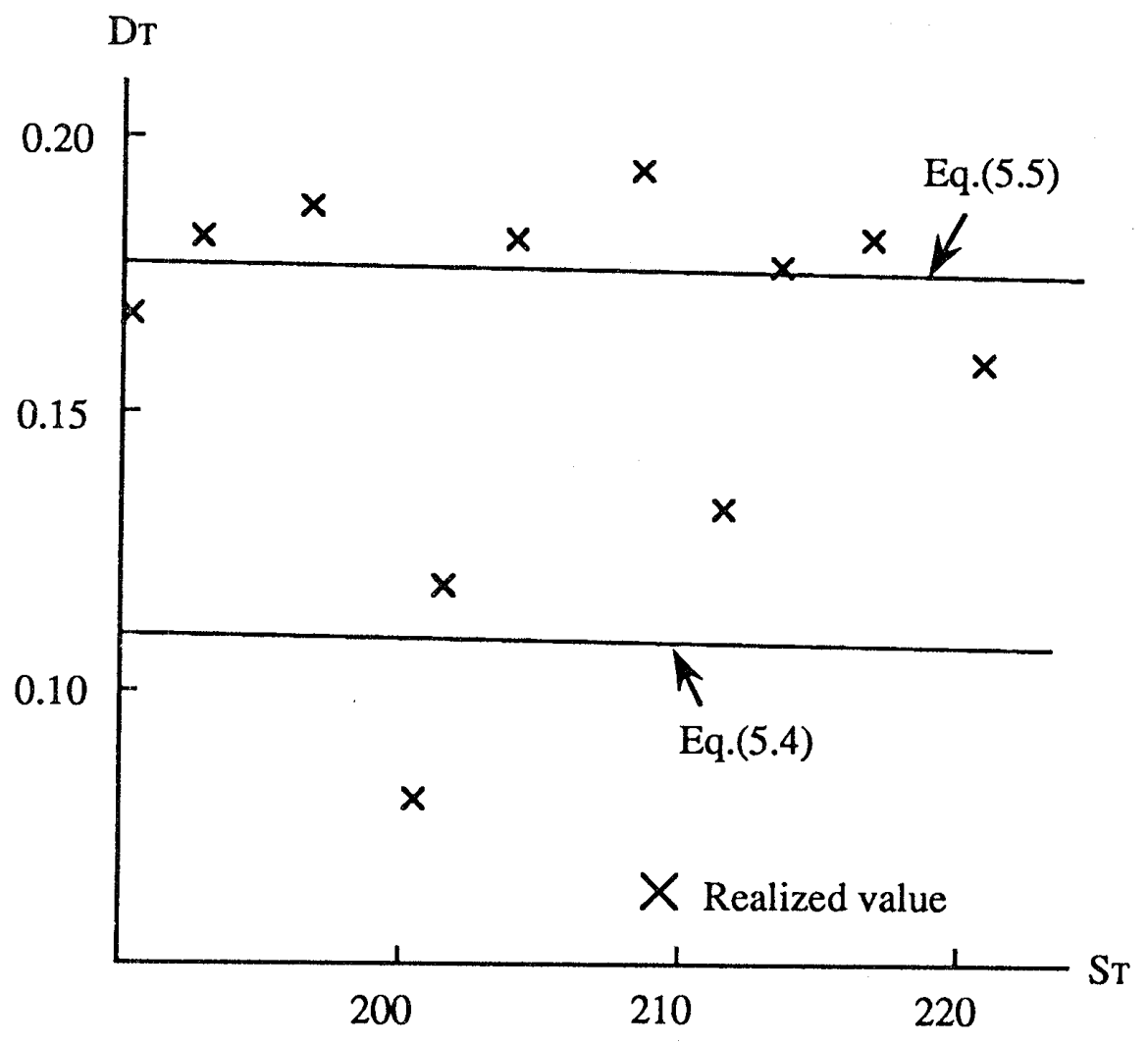

Fig. 6 Realized values and estimates of $D_{T}$

is assumed to be the model of the total growth rate. The growth rate of $X_{T}$ is much less than the rate of $Y_{T}$. Thus, we can let $\left\{m p_{2}-r_{2} y \simeq c\right\}$. Let $\left\{\left(Y_{T+1}-Y_{T}\right) /\left(1000-S_{T}\right)\right\}$ be an explained variable and $\left\{a+b Y_{t}\right\}$ be a regression function. Estimating $a$ and $b$ by the least squared procedure, we can obtain

$$
\left(Y_{T+1}-Y_{T}\right) /\left(1000-S_{T}\right)=0.0026+6.4 \times 10^{-5} Y_{T} .
$$

Let $y_{t}=Y_{T}=0$ and $y_{t+1}=Y_{T+12}$. If we estimate $y_{t+1}$ by

$$
\begin{aligned}
\hat{y}_{t+1} & =y_{t}+12\left(1000-S_{t}\right)\left(0.0026+6.4 \times 10^{-5} y_{t}\right) \\
& =12\left(1000-S_{T}\right) \times 0.0026 \\
& =25, \\
\hat{y}_{t+1} & <Y_{T+12}=30.3 .
\end{aligned}
$$


Considering this,

$$
\begin{aligned}
& \hat{Y}_{T+1}-Y_{T}=\left(0.0032+3 \times 10^{-5} Y_{T}\right)\left(1000-X_{T}-Y_{T}\right) \\
& \hat{y}_{t+1}-y_{t}=\left(0.038+3.6 \times 10^{-4} y_{t}\right)\left(1000-x_{t}-y_{t}\right)
\end{aligned}
$$

From Eqs. (5.3), (5.5), (5.7) and (5.8),

$$
\begin{aligned}
& \hat{X}_{T+1}-X_{T}=\left(2.2 \times 10^{-5} X_{T}-0.0032-0.83 \times{ }^{-5} Y_{T}\right) \\
& \times\left(1000-X_{T}-Y_{T}\right) \\
& \hat{x}_{t+1}-x_{t}=\left(2.6 \times 10^{-4} x_{t}-0.038-10^{-4} y_{t}\right) \\
& \quad \times\left(1000-x_{t}-y_{t}\right)
\end{aligned}
$$

For the purpose of comparison, parameters $a_{i}$ and $b_{i}$ of the most generalized quadratic form

$$
\begin{aligned}
& \hat{X}_{T+1}=X_{T}+a_{0}+a_{1} X_{T}+a_{2} Y_{T}+a_{3} X_{T}^{2}+a_{4} Y_{T}^{2}+a_{5} X_{T} Y_{T} \\
& \hat{Y}_{T+1}=Y_{T}+b_{0}+b_{1} X_{T}+b_{2} Y_{T}+b_{3} X_{T}^{2}+b_{4} Y_{T}^{2}+b_{5} X_{T} Y_{T}
\end{aligned}
$$

parameters $c_{i}$ and $d_{i}$ of the competitive Bass model

$$
\begin{aligned}
& \hat{X}_{T+1}=X_{T}+\left(c_{0}+c_{1} X_{T}+c_{2} Y_{T}\right)\left(m-X_{T}-Y_{T}\right), \\
& \hat{Y}_{T+1}=Y_{T}+\left(d_{0}+d_{1} X_{T}+d_{2} Y_{T}\right)\left(m-X_{T}-Y_{T}\right),
\end{aligned}
$$

and parameters $e_{i}$ and $f_{i}$ of logistic form

$$
\begin{aligned}
& \hat{X}_{T+1}=X_{T}+X_{T}\left(e_{0}+e_{1} X_{T}+e_{2} Y_{T}\right) \\
& \hat{Y}_{T+1}=Y_{T}+Y_{T}\left(f_{0}+f_{1} X_{T}+f_{2} Y_{T}\right)
\end{aligned}
$$

were estimated by the least squares procedure. The estimation error for these equations, where

$$
\epsilon_{X}=\sum\left(\hat{X}_{T}-X_{T}\right)^{2} ; \epsilon_{Y}=\sum\left(\hat{Y}_{T}-Y_{T}\right)^{2}
$$

is shown in Table 1. This shows that Eqs. (5.7) and (5.9) give satisfactory accuracy. In Eq. (5.7), $p_{1}<0$. This value is different from the standard cases in Sec. 4. The paper [14] shows the possibility of negative innovation effect, that is, $\left\{p_{1}<0\right\}$ which results from bottlenecks on the supply side. The same reason may hold true for our example because this service was given for only limited areas. 
Table 1 Estimation errors

\begin{tabular}{|cc|c|c|}
\hline \multirow{2}{*}{ Eq. No } & \multicolumn{1}{|}{} & \\
\cline { 2 - 2 } & $m$ & $\epsilon_{x}$ & $\epsilon_{y}$ \\
\hline & & & \\
\hline$(5.13)$ & 0 & 4.206 & 0.7969 \\
$(5.14)$ & 850 & 4.173 & 0.8335 \\
& 1000 & 4.174 & 0.8315 \\
& 1500 & 4.176 & 0.8283 \\
& & & \\
\hline & & & \\
$(5.11)$ & $(5.12)$ & 3.998 & 0.5003 \\
$(5.7)$ & $(5.19)$ & 358.6 & 0.8162 \\
& $(5.9)$ & 6.157 & 4.559 \\
\hline
\end{tabular}

From Eq. (5.5),

where

$$
s_{t+1}-s_{t}=2.6 \times 10^{-4} s_{t}\left(1000-s_{t}\right)
$$

$$
s_{t}=x_{t}+y_{t}
$$

Differential representation of Eqs. (5.8) and (5.17) is given by

$$
\begin{aligned}
& d s_{t} / d t=2.6 \times 10^{-4} s_{t}\left(1000-s_{t}\right), \\
& d y_{t} / d t=\left(0.038+3.6 \times 10^{-4} y_{t}\right)\left(1000-s_{t}\right) .
\end{aligned}
$$

We can solve Eqs. (5.18) and (5.19) explicitly, differing from the general case of Secs. 3 and 4. The solutions are

$$
\begin{aligned}
& s_{t}=1000 /[1+3.425 \exp \{-0.26(t-1)\}] \\
& y_{t}=0.0746 s_{t}^{1.385}-105.6 \\
& x_{t}=s_{t}-y_{t} .
\end{aligned}
$$

where $s_{1}=226$ and $y_{1}=30$. This result shows that the NCC's share will become larger than NTT's in several years time.

\section{An extension of the Competitive Bass Model}

If $p_{i}, q_{i}$, and $r_{i}$ in Eqs. (2.11) and (2.12) are independent of $m$, the right side of Eq. (3.3) is also independent of $m$. Then, even if $m$ is a function of time, the same relation as that of Eqs. (3.1) and (3.2) holds. However, notice that the time-derivatives of $x$ and $y$ depend on
$m$.

If $m, p_{i}, q_{i}$, and $r_{i}$ in Eqs. (2.11) and (2.12) are constant parameters during each time interval, that is, they are given by step functions of time, the competitive Bass model can
be applied during each time interval. 
Another extension is the introduction of brand switching effect. If the number of subscribers switching brands is proportional to the number of subscribers, Eqs. (2.11) and (2.12) can be corrected by

$$
\begin{aligned}
& d x / d t=(m-x-y)\left(p_{1}+q_{1} x-r_{1} y\right)-e_{1} x+e_{2} y \\
& d y / d t=(m-x-y)\left(p_{2}+q_{2} y-r_{2} x\right)+e_{1} x-e_{2} y
\end{aligned}
$$

In this differential equation system, the same equations as Eqs. (4.4) and (4.5) hold. If $e_{1}>e_{2}$, the number of subscribers of the existing firm is decreased by the competitor. Therefore, if the value, $x_{0}(>0)$, which firm $A$ takes when firm $B$ is going to enter is smaller than $a-b z_{2}$, a similar process to that in case (vi)-(a) in Sec. 4 is experienced at a higher speed than Eqs. (2.11) and (2.12), where $a, b$ and $z_{2}$ are the same quantities as those in Sec. 4. If $x_{0}>a-b z_{2}$ and $e_{1}\left(>e_{2}\right)$ is not larger than $q_{1}$, a similar process to that in case (vi)-(b) in Sec. 4 is experienced, except for the neighborhood of $\{m-x-y=0\}$. (See Fig. 7 where $e_{1}=1, e_{2}=0.1$ and other parameters are the same as Example. 1.) If $x_{0}>a-b z_{2}$ and $e_{1}\left(>e_{2}\right)$ is much larger than $q_{1}$, a similar process to that in case (vi)-(a) rather than that in case (vi)-(b) is experienced. (See Fig. 8 where $e_{1}=30, e_{2}=3$ and other parameters are the same as Example. 1.)

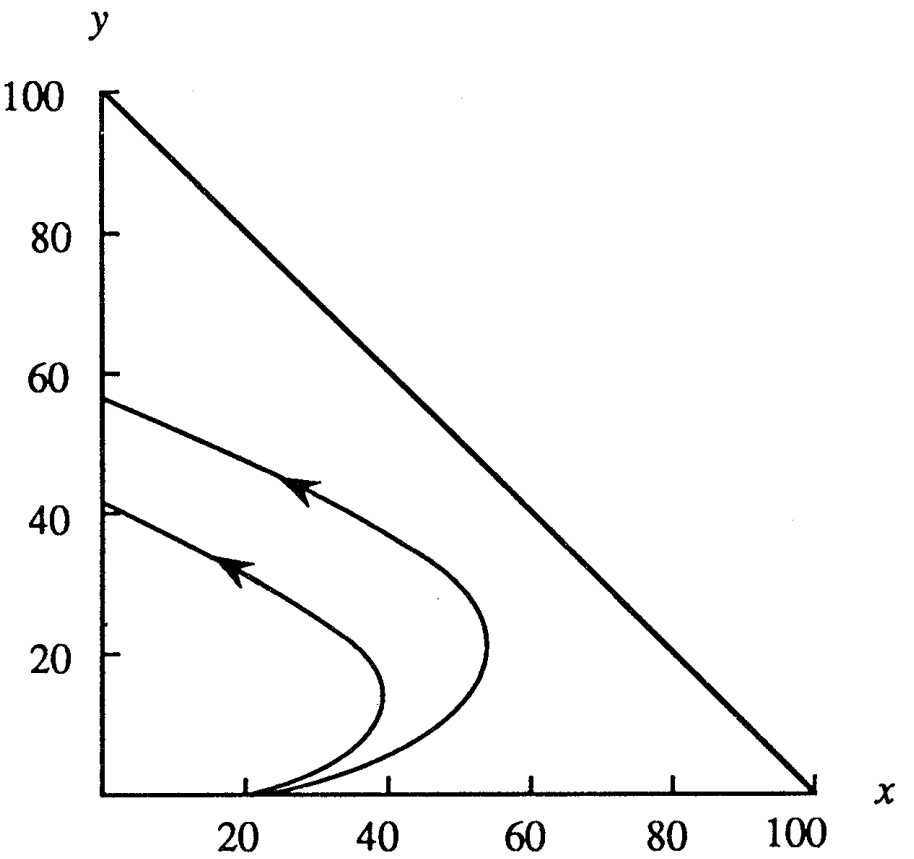

Fig. 7 Diffusion process (1) of Eqs. (6.1) and (6.2) 


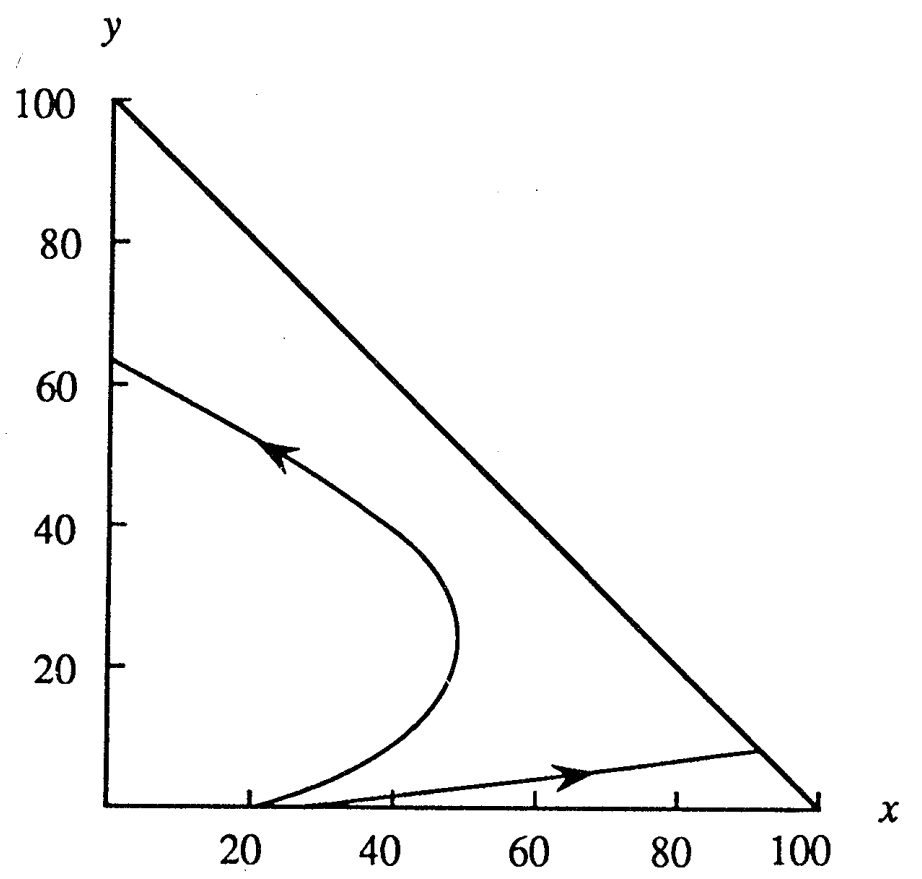

Fig. 8. Diffusion process (2) of Eqs. (6.1) and (6.2)

Singular points in Eqs. (2.11) and (2.12) are given by

$$
\{m-x-y=0\} \text { and }\{x=a, y=b\},
$$

but a singular point in Eqs. (6.1) and (6.2) is given by a crosspoint of two second-order curves relating to $a$ and $b$ when the coordinate of the singular point is $(a, b)$. The stability around the singular point can be discussed in the same way as in Sec. 4 by approximating linear system. However, since discussion using simple formulae may be impossible, it will not be mentioned further.

\section{Conclusion}

This paper proposed a competitive Bass model and showed its diffusion process. Bass [3] stressed the importance of simplicity, and this paper preserves the simplicity. However, to select good sales strategies according to forecasts of future demand, variables such as advertising cost, sales promotion cost and price should be represented in the model, thus the parameters $p_{i}, q_{i}$ and $r_{i}$ should be related to marketing mix strategies. In this paper, the parameter $m$, which is the total amount of purchase during the period considered, is a constant, but models in which $m$ varies with time or market extension are being studied by the author. Another defect in this paper is insufficient discussion of the case $\{d x / d t<0$ or $d y / d t<0\}$ and the brand switching effect. To consider the brand switching effect in detail, modeling different from Eqs. (6.1) and (6.2) may be needed.

Telecommunication services in Japan have only recently experienced competition and thus there is not sufficient data to show the effectiveness of the competitive Bass model or the need for more sophisticated models. We hope readers will discuss the merits and demerits of the competitive Bass model in practical applications.

Acknowledgement. The author is very grateful to the referees for their invaluable comments and suggestions. 


\section{References}

[1] Bartlett, M.S.: On Theoretical Models for Competitive and Predatory Biological Systems. Biometrika, Vol. 44, (1957), 27-42.

[2] Bass, F.M.: A New Product Growth for Model Consumer Durables. Management Science, Vol. 15, No. 5 (1969), 215-227.

[3] Bass, F.M.: The Adoption of a Marketing Model: Comments and Observations. Innovation Diffusion Models of New Product Acceptance (V. Mahajan and Y. Wind), Ballinger, Massachusetts (1986), 27-33.

[4] Dockner, E. and JØrgensen, S.: Optimal Advertising Policies for Diffusion Models of New Product Innovation in Monopolistic Situations. Management Science, Vol. 34, No. 1 (1988), 119-130.

[5] Dolan, R.J. and Jeuland, A.P.: Experience Curves and Dynamic Demand Models: Implications for Optimal Pricing Strategies. Journal of Marketing, Vol. 45, (1981), 52-62.

[6] Dolan, R.J., Jeuland, A.P. and Muller, E.: Models of New Product Diffusion: Extension to Competition against Existing and Potential Firms over time. Innovation Diffusion Models of New Product Acceptance (V. Mahajan and Y. Wind), Ballinger, Massachusetts (1986), 117-149.

[7] Eliashberg, J. and Jeuland, A.P.: The Impact of Competitive Entry in a Developing Market upon Dynamic Pricing Strategies. Marketing Science, Vol. 5, No. 1 (1986), 20-36.

[8] Feichtinger, G.: Optimal Pricing in a Diffusion Model with Concave Price-Dependent Market Potential. Operations Research Letters, Vol. 1, No. 6 (1982), 236-240.

[9] Haberman, R.: Mathematical Model: Population Dynamics. Prentice Hall, NJ, 1977.

[10] Horsky, D. and Simon, L.S.: Advertising and the Diffusion of New Products. Marketing Science, Vol. 2, No. 1 (1983), 1-17.

[11] Kalish, S. and Lilien, G.L.: A Market Entry Timing Model for New Technologies. Management Science, Vol. 32, No. 2 (1986), 194-205.

[12] Kaplan, W.: Ordinary Differential Equations. Addison-Wesley, Massachusetts, 1958.

[13] Mahajan, V. and Peterson, R.A.: Innovation Diffusion in a Dynamic Potential Adopter Population. Management Science, Vol. 24, No. 15 (1978), 1589-1597.

[14] Robinson, B. and Lakhani, C.: Dynamic Price Models for New-Product Planning. Management Science, Vol. 21, No. 10 (1975), 1113-1122.

[15] Simon, H. and Sebastian, K.-H.: Diffusion and Advertising: The German Telephone Campaign. Management Science, Vol. 33, No. 4 (1987), 451-466.

Tohru Ueda:

NTT Communication

Switching Laboratories, 9-11

Midori-Cho 3-Chome Musashino-Shi,

Tokyo, 180, Japan. 